\title{
Cyclin-Dependent Kinase 5 Phosphorylates Disabled 1 Independently of Reelin Signaling
}

\author{
Lakhu Keshvara, Susan Magdaleno, David Benhayon, and Tom Curran \\ Department of Developmental Neurobiology, St. Jude Children's Research Hospital, Memphis, Tennessee 38105
}

Two major signaling pathways that control neuronal positioning during brain development have been uncovered as a result of genetic and biochemical studies on neurological mouse mutants. Mice deficient in Reelin, Disabled 1 (Dab1), or both the very low-density lipoprotein receptor (VLDLR) and the apolipoprotein E receptor 2 (ApoER2) exhibit identical neuroanatomic defects in laminar structures throughout the brain. These proteins function as components of the Reelin signaling pathway. Reelin is a secreted glycoprotein that binds to VLDLR and ApoER2, inducing tyrosine phosphorylation of Dab1, an intracellular adapter protein. Neuronal migration is also regulated by cyclin-dependent kinase 5 (Cdk5) and its activating subunits p35 and p39. Mice deficient in Cdk5, p35, or both p35 and p39 exhibit lamination defects that are similar but not identical to those observed in mice with a defect in the Reelin signaling pathway. Cdk5 phosphorylates proteins that maintain cytoskel-

Analysis of neurological mutant mice has led to the identification of several genes that are critical for neuronal positioning during development of the mammalian brain (Rice and Curran, 2001). One of the most widely studied neurological mutants is the ataxic mouse reeler, which carries a disruption in the gene encoding Reelin, a large secreted glycoprotein (D'Arcangelo et al., 1995). In the absence of Reelin, neurons fail to assume correct positions within the developing brain, resulting in severe defects in lamination of brain structures, including the cerebral cortex, cerebellum, and hippocampus (for review, see D'Arcangelo and Curran, 1998). Identical defects were later described in mice deficient in Disabled 1 (Dab1), or both very low-density lipoprotein receptor (VLDLR) and apolipoprotein E receptor 2 (ApoER2), providing strong evidence that these proteins participate in the Reelin signaling pathway (Howell et al., 1997b; Sheldon et al., 1997; Trommsdorff et al., 1999). Mice lacking cyclin-dependent kinase $5(\mathrm{Cdk} 5), \mathrm{p} 35$, or both p35 and p39 exhibit lamination defects that are similar but not identical to those observed in reeler (Ohshima et al., 1996; Chae et al., 1997; Gilmore et al., 1998; Kwon and Tsai, 1998; Ko et al., 2001). In contrast to the situation in reeler,

\footnotetext{
Received Feb. 8, 2002; revised April 1, 2002; accepted April 1, 2002.

This work was supported in part by National Institutes of Health Cancer Center Support CORE Grant P30 CA21765, National Institutes of Health/National Institute of Neurological Disorders and Stroke Grant RO1-NS36558 (T.C.), the American Lebanese Syrian Associated Charities, and Human Frontiers Science Program Grant RG67/98. We thank Dr. Ashok Kulkarni for the Cdk5 mutant mice, Dr. Li-Huei Tsai for Cdk5 and p35 expression plasmids, Dr. Jonathan Cooper for B3 anti-Dab1 antibodies, and Dr. Hee-Won Park for purified His-tagged Dab1. We are also thankful to Dr. Li-Huei Tsai and Dr. Janet Volker for helpful discussions.

Correspondence should be addressed to Dr. Tom Curran, Department of Developmental Neurobiology, St. Jude Children's Research Hospital, 332 North Lauderdale, Memphis, TN 38105. E-mail: fos1@aol.com.

Copyright (ㄷ) 2002 Society for Neuroscience $0270-6474 / 02 / 224869-09 \$ 15.00 / 0$
}

etal structures and promote cell motility. To explore the possibility that Cdk5 influences the Reelin pathway, we sought to determine whether Dab1 is a substrate for Cdk5. Here we show that Cdk5 phosphorylates Dab1 on serine 491 in vitro and in vivo, independently of Reelin signaling. We also show that ectopic neurons in Cdk5-deficient mice exhibit reduced levels of Reelin signaling during later stages of cortical development, although Cdk5 is not required for Reelin-induced tyrosine phosphorylation of Dab1. Although the functional significance of Dab1 serine phosphorylation is unclear, our results suggest that there is biochemical cross-talk between two signaling pathways that control cell positioning.

Key words: reeler; neuronal migration; Reelin; VLDLR; ApoER2; Dab1; Cdk5; p35; p39; phosphorylation; signal transduction

initial waves of migrating neurons in $C d k 5^{-1-}$ mice successfully split the preplate, but subsequent migration failures result in inversion of layers beneath an ectopic subplate. Therefore, Cdk5 is thought to be a component of a signaling pathway distinct from the Reelin pathway.

The phosphotyrosine-binding (PTB) domain of Dab1 associates with an NPxY motif in the cytoplasmic region of VLDLR and ApoER2 (Trommsdorff et al., 1998). Reelin binds to these receptors on the surface of neurons, triggering tyrosine phosphorylation of Dab1 (D'Arcangelo et al., 1999; Hiesberger et al., 1999; Howell et al., 1999a). In the absence of Reelin or the receptors, Dab1 accumulates in a hypophosphorylated form (Rice et al., 1998; Trommsdorff et al., 1999). Tyrosine phosphorylated Dab1 may couple Reelin signaling to downstream molecular machinery involved in cell positioning. Thus, Dab1 could serve as a nexus for numerous signaling pathways.

Cdk5 is a serine-threonine kinase that is ubiquitously expressed, but its catalytic activity is dependent on the neuronal regulators p35 or p39 (Tsai et al., 1993, 1994; Lew et al., 1994; Tang et al., 1995). Cdk5 phosphorylates a variety of substrates in vitro, including proteins known to play a role in cell adhesion and migration (for review, see Dhavan and Tsai, 2001). One possible way in which $\mathrm{Cdk} 5$ can influence Reelin signaling is through phosphorylation of Dab1. We showed previously that Dab1 is phosphorylated predominantly on serine-threonine residues in a transient transfection system (Homayouni et al., 1999). Indeed, Dab1 contains numerous potential phosphorylation sites for Cdk5. Here we show that Cdk5 phosphorylates serine 491 of Dab1 in vitro, as well as in vivo. Unlike tyrosine phosphorylation, serine phosphorylation of Dab1 occurs independently of Reelin signaling. Furthermore, $\mathrm{Cdk} 5$ is not required for Reelin-induced 
tyrosine phosphorylation of Dab1. Thus, although these phosphorylation events are governed by two distinct pathways, our results raise the possibility that $\mathrm{Cdk} 5$ modulates Reelin signaling downstream of Dab1.

\section{MATERIALS AND METHODS}

Materials. The Dab1-hemagglutinin (Dab1-HA) expression plasmid and the glutathione $S$-transferase (GST) fusion constructs containing fulllength Dab1 or Dab1 domains have been described previously (Keshvara et al., 2001). Cdk5 and p35 expression plasmids were provided by Dr. Li-Huei Tsai (Harvard Medical School, Boston, MA). Site-directed mutagenesis was conducted using the QuikChange site-directed mutagenesis kit (Stratagene, La Jolla, CA). Purified polyhistidine-tagged Dab1 (Dab1-His) was provided by Dr. Hee-Won Park (St. Jude Children's Research Hospital). Anti-Cdk5 antibodies were obtained from Santa Cruz Biotechnology (Santa Cruz, CA). Rabbit anti-Dab1 antibodies (CT38) raised against the $\mathrm{C}$ terminal region of Dab1 and goat anti-Dab1 PTB have been described previously (Keshvara et al., 2001). B3 antiDab1 antibodies were a gift from Dr. Jonathan Cooper (Fred Hutchinson Cancer Research Center, Seattle, WA).

Phosphopeptide antibodies. Synthesis of the phosphopeptide PSer491 [TPAPRQSS $\left(\mathrm{PO}_{4}\right)$ PSKSSA] and chemical coupling to KLH or Sepharose were performed by Hartwell Center for Bioinformatics and Biotechnology (St. Jude Children's Research Hospital). Immunization and antibody production were performed by Rockland (Gilbertsville, PA). Antisera were screened by Western blotting using lysates of human embryonic kidney 293 (HEK293T) cells transfected with Dab1 and $\mathrm{Cdk} 5 / \mathrm{p} 35$.

Antibody purification. To purify anti-PSer491, $250 \mu \mathrm{l}$ of antiserum was diluted with PBS and passed several times through a column containing $500 \mu \mathrm{l}$ of PSer491-Sepharose slurry ( $\sim 5 \mathrm{mg}$ of phosphopeptide). After washing the column with PBS, bound antibodies were eluted with $100 \mathrm{~mm}$ glycine, $\mathrm{pH}$ 2.8. The eluate was neutralized with $1 \mathrm{~m}$ Tris- $\mathrm{HCl}, \mathrm{pH}$ 9, and dialyzed against PBS. For the purification of anti-Dab1 PTB antibodies, $10 \mathrm{mg}$ of purified GST-PTB was coupled to Sepharose using AminoLink Plus Immobilization kit (Pierce, Rockford, IL). Goat anti-PTB antisera were diluted with PBS and passed through this column several times, and the bound antibodies were eluted as above. The antibodies were further purified by passing once over GST-Sepharose column (Pierce).

Protein expression. GST fusion proteins were expressed in BL21 bacterial strains (Stratagene) as described previously (Keshvara et al., 2001). Briefly, bacteria were grown in LB media and induced with $100 \mu \mathrm{M}$ $\beta$-D-galactopyranoside for $4 \mathrm{hr}$ at $37^{\circ} \mathrm{C}$. Bacterial pellets were lysed by sonicating in PBS containing 1\% Triton X-100, 5 mm EDTA, 2 mm phenylmethylsulfonyl fluoride, $40 \mu \mathrm{g} / \mathrm{ml}$ aprotinin, and $40 \mu \mathrm{g} / \mathrm{ml}$ leupeptin. The lysates were incubated with glutathione-Sepharose (Amersham Biosciences, Piscataway, NJ) at $4^{\circ} \mathrm{C}$. Bound proteins were eluted with 15 $\mathrm{mm}$ glutathione and dialyzed in $50 \mathrm{~mm}$ Tris-HCl, $\mathrm{pH}$ 7.5.

Animal genotyping and dissection. Reeler, Cdk5 mutant, and wild-type mice were used. Conception was determined by the presence of a vaginal plug, with the day of conception considered to be embryonic day 0 (E0). Cdk5 mutant embryos and wild-type littermates were generated by crossing $C d k 5$ heterozygotes. Genotyping was accomplished by PCR using the following oligonucleotide primers: 5 '-tcatgagattgtggctctgaag; 5'-tcttgtcactatgcaggacatc; and 5 -gcatgctccagactgcttg. Embryonic and adult brains were removed by dissection and were either snap frozen in liquid nitrogen or triturated for tissue culture. Reeler embryos were generated by crossing homozygous reeler mice.

Immunohistochemical analysis. Embryos from E15.5 to E17.5 were delivered surgically and perfused intracardially with $4 \%$ paraformaldehyde in $0.1 \mathrm{M} \mathrm{PBS}, \mathrm{pH}$ 7.2. The tissue was postfixed for $4 \mathrm{hr}$ at $4^{\circ} \mathrm{C}$, cryoprotected in $25 \%$ sucrose, embedded in TBS tissue freezing medium (Triangle Biomedical Sciences, Durham, NC) on dry ice, and processed using standard techniques (Rice et al., 1998). For all experiments, control and test tissues were embedded together in one block to minimize differences in section thickness. Colocalization of Cdk5 and Dab1 was performed using rabbit anti-Cdk5 antibodies (1:100) (Santa Cruz Biotechnology) and goat anti-Dab1 antibodies (1:100). Nonspecific antibody binding was blocked with $2.5 \%$ BSA in PBS, pH 7.3, containing $0.01 \%$ Triton X-100 (PBST) for $1 \mathrm{hr}$ at room temperature. For double staining, goat anti-Dab1 and rabbit anti-Cdk5 antibodies were diluted together in BSA-PBST and incubated with tissue sections at $4^{\circ} \mathrm{C}$ overnight. Tissue sections were washed thoroughly in PBS and then incubated with AlexaFluor 594-conjugated donkey anti-goat IgG (1:200) and AlexaFluor 488-conjugated goat anti-rabbit IgG (1:200) (Molecular Probes, Eugene, OR) for $1 \mathrm{hr}$ at room temperature. Sections were washed as described above, and coverslips were mounted with Vectashield Mounting Medium (Vector Laboratories, Burlingame, CA). Adjacent sections identically processed without the primary antibody did not show any significant staining above background (data not shown).

Embryonic day 18.5 sagittal sections from $\mathrm{Cdk} 5^{+/+}$and $\mathrm{Cdk} 5^{-1-}$ brains were incubated in a humid chamber overnight at $4^{\circ} \mathrm{C}$ with rabbit anti-Dab1 antibodies (B3; 1:800). Rabbit anti-Dab1 antibodies were detected using the Vectastain Elite ABC kit (Vector Laboratories) as described previously (Rice et al., 1998). $\mathrm{Cdk} 5^{+/+}$and $\mathrm{Cdk} 5^{-/-}$tissues were cut and slide mounted in the same tissue block and incubated for equivalent times in diaminobenzidine (DAB) substrate (Kirkegaard \& Perry, Gaithersburg, MD). As negative controls, adjacent sections from the same tissue block were identically processed without the primary antibody, and no DAB reaction product was observed (data not shown). Slides were dehydrated and mounted by standard techniques. Immunofluorescence and immunohistochemical detection of Dab1 and Cdk5 were performed using an Olympus BX60 upright microscope, and images were acquired with a Hamamatsu (Bridgewater, NJ) C5810 video camera and imported directly into Adobe Photoshop 5.0 (Adobe Systems, San Jose, CA). Images of Dab1 and Cdk5 double staining of postnatal day 7 (P7) cerebellum were acquired using a Leica (Nussloch, Germany) TCS confocal laser scanning microscope equipped with a $100 \times$ oil objective. Contrast and brightness were applied equally to each figure to obtain high-quality photomicrographs.

Reelin stimulation and brain lysates. Stimulation of embryonic neurons with purified Reelin has been described previously (Keshvara et al., 2001). Briefly, neurons obtained by trituration of embryonic brains were treated with either purified recombinant Reelin or a control supernatant for $15 \mathrm{~min}$ at $37^{\circ} \mathrm{C}$. After incubation, neurons were either snap frozen or lysed immediately. Reelin-treated neuronal pellets were lysed in cell lysis buffer (25 mm Tris-HCl, $1 \%$ Nonidet P-40, $150 \mathrm{~mm} \mathrm{NaCl}, 5 \mathrm{~mm}$ EDTA, $1 \mathrm{~mm}$ sodium orthovanadate, $20 \mathrm{~mm}$ sodium fluoride, and $20 \mu \mathrm{g} / \mathrm{ml}$ each of aprotinin and leupeptin). Whole brains were also homogenized in this lysis buffer. Cell lysates as well as brain homogenates were clarified by centrifugation at $10,000 \times g$ at $4^{\circ} \mathrm{C}$ for $20 \mathrm{~min}$.

Cell culture and transfection. HEK293T cells (American Type Culture Collection, Manassas, Virginia) were maintained in DMEM (BioWhittaker, Walkersville, MD) supplemented with $10 \%$ fetal bovine serum (BioWhittaker), $10 \mathrm{U} / \mathrm{ml}$ penicillin-streptomycin mixture (BioWhittaker), and 2 mM GlutaMAX (Invitrogen, Carlsbad, CA). Transfections were performed using FuGene 6 transfection reagent (Roche Molecular Biochemicals, Indianapolis, IN). Twenty-four hours after transfection, cells were lysed in cell lysis buffer.

Immunoprecipitation and Western blotting. Cell or brain lysates were incubated with anti-Cdk5 or anti-Dab1 antibodies for $4^{\circ} \mathrm{C}$ for $2 \mathrm{hr}$. Immune complexes were collected with protein G-agarose beads (Pierce) and washed three times with cell lysis buffer. Immunoprecipitates were boiled in SDS sample buffer, separated by SDS-PAGE, and blotted to nitrocellulose membranes. Nitrocellulose membranes were blocked with $5 \%$ nonfat dry milk in TBST (50 mM Tris, $\mathrm{pH} 7.4,150 \mathrm{~mm} \mathrm{NaCl}$, and $1 \%$ Tween 20) for $1 \mathrm{hr}$ at room temperature. The following antibodies were used for various immunoblots: anti-PSer491 (1:1000), anti-Cdk5 (1:2000), anti-PY198 (1:100), 4G10 (1:2000) (Upstate Biotechnology, Lake Placid, NY), and anti-Dab1 (CT38; 1:5000). All dilutions were made in blocking solution, and incubations were performed at room temperature for $1 \mathrm{hr}$. The membranes were washed in TBST and incubated with horseradish peroxidase-conjugated secondary antibody (1: $10,000)$ for $1 \mathrm{hr}$. Detection was performed using Super Signal West Dura Extended Duration Substrate (Pierce).

In vitro kinase assay. For kinase assays, anti-Cdk5 immunoprecipitates were washed three times with cell lysis buffer and once with $25 \mathrm{~mm}$ Tris- $\mathrm{HCl}, \mathrm{pH}$ 7.5. The beads were then incubated with a kinase reaction mixture containing $5 \mu \mathrm{g}$ of substrate (GST fusions or Dab1-His), $10 \mathrm{~mm}$ $\mathrm{MgCl}_{2}$, and $10 \mu \mathrm{Ci}$ of ATP $(10 \mu \mathrm{M})$ in $25 \mathrm{~mm}$ Tris-HCl, $\mathrm{pH}$ 7.5. The reactions were performed at $30^{\circ} \mathrm{C}$ for $15 \mathrm{~min}$ and then stopped by boiling in SDS sample buffer. Phosphorylated proteins were separated by SDSPAGE, transferred to nitrocellulose membranes, and detected by autoradiography. Membranes were stained with Coomassie brilliant blue to locate the proteins before trypsinization.

Phosphopeptide mapping. Phosphoproteins were excised from nitrocellulose membranes and digested with trypsin (Promega, Madison, WI) as described previously (Keshvara et al., 2001). Phosphopeptides were dried and separated by electrophoresis on an alkaline $40 \%$ polyacrylamide gel 

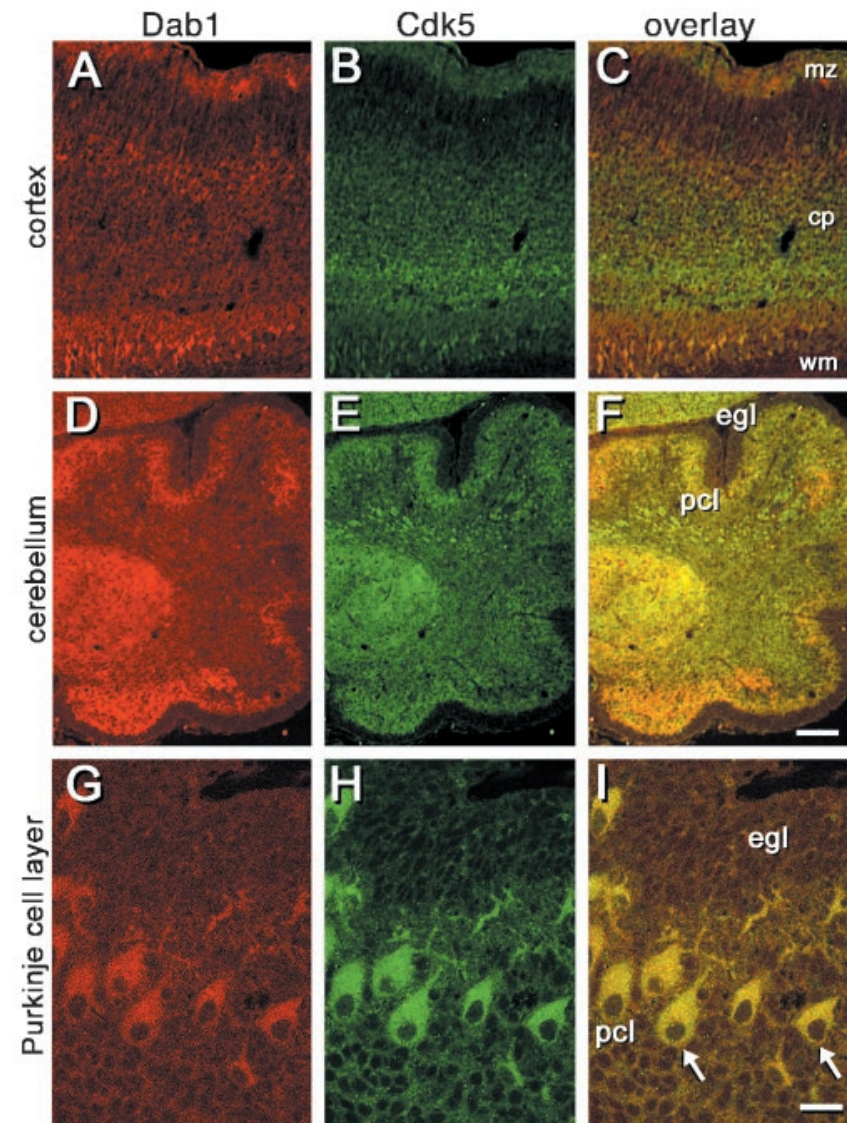

Figure 1. Dab1 and Cdk5 display distinct but overlapping expression patterns in the cerebral cortex and cerebellum at postnatal day 0. A, Dab1 (red) is present in the cell bodies and dendritic processes of neurons in the cerebral cortex. $B, \mathrm{Cdk} 5$ ( green) is detected in postmitotic neurons in the cortical plate and in the thalamocortical fibers. $C$, Double staining shows that Dab1 and Cdk5 expression overlap in postmitotic neurons in the cerebral cortex. $D$, In the cerebellum, Dab1 is expressed in Purkinje cells as they migrate to form the Purkinje cell layer. E, Cdk5 is expressed in Purkinje cells, but it is also present in cerebellar nuclei and in axon tracts in the cerebellum. F, Double staining shows that Dab1 and Cdk5 overlap in the Purkinje cells of the developing cerebellum (yellow). Scale bar (in $F$ ), $\sim 100 \mu \mathrm{m}$. $G$, Dab1 is present in Purkinje cell bodies and dendrites in the postnatal day 7 cerebellum. $H, \mathrm{Cdk} 5$ is also present in Purkinje cell bodies and dendrites. $I$, Double staining in the same tissue section shows the overlap of Dab1 and Cdk5 expression in Purkinje cells. Arrows point out individual Purkinje cells in the overlay image. Scale bar (in $I$ ), $\sim 10$ $\mu \mathrm{m} . m z$, Marginal zone; $c p$, cortical plate; $w m$, white matter; egl, external germinal layer; $p c l$, Purkinje cell layer.

as described previously (Keshvara et al., 2001). After the tracking dye had migrated to $R_{\mathrm{F}}=0.5$, the gel was dried, and phosphopeptides were detected by autoradiography.

\section{RESULTS}

\section{Dab1 and Cdk5 are coexpressed in the developing brain}

Mice that lack Dab1 or Cdk5 exhibit defects in the migration patterns of neurons in the cortical plate and in the cerebellum. However, the defective migration of neurons in Cdk5-deficient mice is distinct from the abnormal migrations observed in Dab1deficient mice. During early corticogenesis, Dab1 is present in the cells that occupy the ventricular zone during the preplate stage of development, and later it is present in migrating neurons in the intermediate zone and in neurons within the cortical plate (Fig. 1A) (Rice et al., 1998). Cdk5 is detected as early as E14.5 in postmitotic neurons in the cerebral cortex (Tsai et al., 1993), and it is predominantly present in thalamocortical tracts (Fig. $1 B$ ). In P0 cerebellum, Dab1 is expressed in Purkinje cells as they migrate to form the Purkinje cell layer (Fig. 1D). Cdk5 is expressed in Purkinje cells in the cerebellum, but it is also present in large cells of the cerebellar cortex, such as the basket, Lugaro, or Golgi cells (Ohshima et al., 1999) (Fig. 1E). Confocal microscopy of postnatal day 7 cerebellum shows expression of both Dab1 and Cdk5 within cell bodies and dendrites of individual Purkinje cells as they migrate to form the Purkinje cell layer in the developing cerebellum (Fig. 1G-I). Therefore, because these analyses show that Dab1 and Cdk5 are present in overlapping cell populations in the cerebral cortex and in Purkinje cells of the cerebellum (Fig. $1 C, F, I)$, it is possible that $\mathrm{Cdk} 5$ phosphorylates Dab1 directly in vivo.

\section{Cdk5 Phosphorylates Dab1 in vitro}

To determine whether $\mathrm{Cdk} 5$ can phosphorylate Dab1 in vitro, an active Cdk5-p35 complex was immunoprecipitated from brain homogenates and used to phosphorylate recombinant Dab1-His. Indeed, Dab1 was highly phosphorylated by anti-C $\mathrm{dk} 5$ immunoprecipitated from brain extracts of wild-type mice (Fig. $2 A$ ). In contrast, immunoprecipitates from brain extracts of $\mathrm{Cdk} 5$ deficient littermates did not yield any significant kinase activity.

To begin to localize the Cdk5 phosphorylation sites on Dab1, we repeated the in vitro Cdk5 kinase assays using GST fusion proteins representing three distinct regions of Dab1, namely, the PTB domain (GST-PTB; residues 1-179), the middle region (GST-Mid; residues 180-399), and the C-terminal region (GSTCT; residues 400-555). As shown in Figure 2A, fusion proteins containing the PTB domain and the middle region of Dab1 were not phosphorylated to any significant extent. In contrast, the GST fusion product containing the C-terminal region of Dab1 was phosphorylated to almost the same extent as full-length Dab1. Therefore, the major sites of in vitro phosphorylation by $\mathrm{Cdk} 5$ are likely to be confined to the $\mathrm{C}$ terminus of Dab1. To confirm this observation, we generated tryptic phosphopeptides from in vitro phosphorylated Dab1-His and GST-CT and analyzed them by electrophoretic separation on a $40 \%$ acrylamide gel. As shown in Figure $2 B$, two major phosphopeptides, phosphopeptides 1 and 2, were clearly resolved, and both were present in the C-terminal region of Dab1.

Cdk5 exhibits a strong preference for serine and threonine residues with an adjacent proline and a basic amino acid at the third residue (Ser/Thr-Pro-X-Arg/Lys) (Songyang et al., 1996). Although there are several Ser-Pro/Thr-Pro sequences present within the C-terminal domain of Dab1, only two sites, serines 491 and 515 , correspond to the consensus sequence preferred by Cdk5 (Fig. 2C). Both serine 491 and serine 515 are followed by proline in the +1 position and lysine in the +3 position. Serine 491 is located within a small tryptic fragment (QSSPSK) comprised of only six amino acids, representing the smallest possible tryptic peptide that would contain a Cdk5 phosphorylation site within the $\mathrm{C}$-terminal domain of Dab1 (Fig. 2C). Therefore, it is highly probable that phosphopeptide 2 resulted from in vitro phosphorylation of serine 491. The other putative site for Cdk5 phosphorylation, serine 515, is contained within a much larger tryptic fragment (SSASHVSDPTADDIFEEGFESPSK). Phosphopeptide 1 is likely a result of phosphorylation of serine 515 within this tryptic fragment. Therefore, our phosphopeptide mapping analysis was consistent with the hypothesis that C dk5 preferentially phosphorylated serines 491 and 515 in vitro. 
A

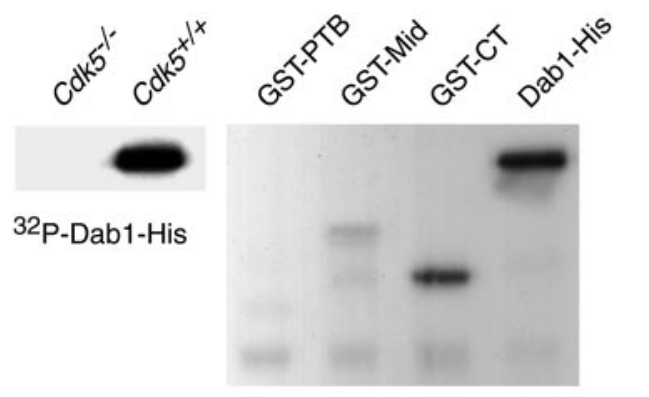

B

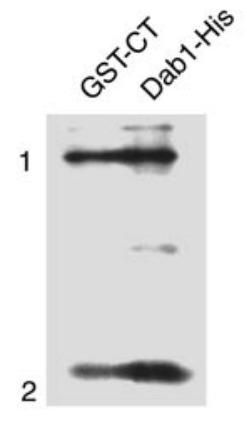

C

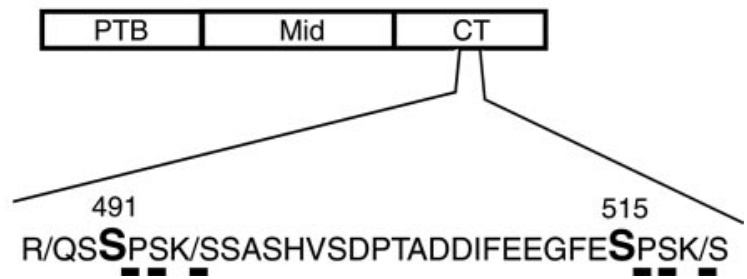

Figure 2. Cdk5-p35 immunoprecipitated from brain phosphorylates Dab1 in vitro. $A$, The Cdk5-p35 complex was immunoprecipitated from detergent lysates of either $C d k 5^{-/-}$or $C d k 5^{+/+}$P0 brains. The immunoprecipitates were then used as source of kinase activity to phosphorylate either full-length Dab1-His or GST fusion proteins containing Dab1 domains (GST-PTB, residues 1-179; GST-Mid, residues 180-399; GST$\mathrm{CT}$, residues $400-555)$ in the presence of $\left[\gamma_{-}{ }^{32} \mathrm{P}\right] \mathrm{ATP}$ in vitro. Proteins were separated by SDS-PAGE, transferred to nitrocellulose, and located by autoradiography. $B$, In vitro phosphorylated GST-CT and Dab1-His (from $A$ ) were trypsinized off the membrane, and tryptic fragments were resolved by alkaline $40 \%$ polyacrylamide gel electrophoresis. Autoradiography revealed two major phosphopeptides (phosphopeptides 1 and 2). $C$, Schematic representation of Dab1 domains. The $\mathrm{C}$-terminal region of Dab1 (CT) gives rise to two phosphopeptides as a result of phosphorylation by Cdk5-p35. Two serines, serine 491 and serine 515, contain the consensus sequence for $\mathrm{Cdk} 5$ phosphorylation, including proline in +1 position and lysine residue in +3 position.

\section{Anti-PSer491 antibodies recognize Cdk5-catalyzed Dab1 phosphorylation}

We recently used phosphopeptide-specific antibodies to identify Reelin-induced sites of tyrosine phosphorylation in Dab1 (Keshvara et al., 2001). Therefore, we adopted the same approach to determine whether Dab1 is phosphorylated by $\mathrm{Cdk} 5$ in vivo. Although our phosphopeptide mapping analysis suggested that both serines 491 and 515 are in vitro sites of Cdk5 phosphorylation, we consistently observed that serine 491 was more highly phosphorylated. Therefore, we chose to generate antibodies
A

B

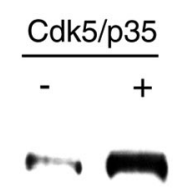

PSer491

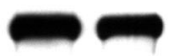

Dab1

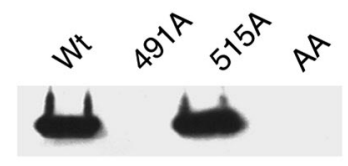

PSer491

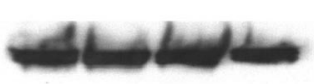

Dab1
Figure 3. Characterization of anti-PSer491 phosphopeptide-specific antibodies. $A$, HEK293T cells were transfected with either Dab1-HA alone $(-)$ or Dab1-HA plus both Cdk5 and p35 (+). Lysates from the transfected cells were separated by SDS-PAGE, transferred to nitrocellulose, and probed with antibodies raised against a phosphopeptide encompassing PSer491. To confirm the presence of Dab1, the membrane was stripped and reprobed with anti-Dab1 antibodies. $B$, Wild-type Dab1-HA $(W t)$ or mutant forms of Dab1 carrying alanine substitutions at residues $491(491 A), 515(515 A)$, or both 491 and $515(A A)$ were transiently expressed in HEK293T cells along with Cdk5 and p35. The site-specificity of the antibodies was assessed by Western blotting of the resulting lysates.

against a phosphopeptide corresponding to this phosphorylation site. The efficacy of the resulting antisera was determined by Western blotting using lysates from HEK293T cells transiently transfected with either Dab1 alone or in the presence of $\mathrm{Cdk} 5$ and p35. As shown in Figure $3 A$, the antiserum preferentially recognized Dab1 when it was cotransfected with Cdk5 and p35, suggesting that the antibodies specifically reacted with phosphorylated Dab1. To confirm the specificity of the antibodies, we generated a mutant of Dab1 in which serine 491 was substituted with an alanine residue (491A). The antibodies also failed to recognize this mutant protein, as well as a combination mutant in which both serine 491 and serine 515 of Dab1 were converted to alanine (AA) (Fig. 3B). In contrast, substitution of serine 515 alone (515A) with alanine did not have any effect on antibody recognition. Western blots with anti-Dab1 antibodies confirmed that the mutant forms of Dab1 were expressed at equivalent levels. Therefore, these results indicate that $\mathrm{Cdk} 5$ phosphorylates Dab1 on serine 491 in transfected cells and that the antibodies are highly specific for this phosphoserine site.

\section{Cdk5-dependent in vivo phosphorylation of Dab1 on serine 491}

To determine whether serine 491 is phosphorylated in vivo, Dab1 was immunoprecipitated from brain extracts prepared from E16.5 $C d k 5^{-/-}, C d k 5^{+/-}$, or $C d k 5^{+/+}$embryos, and Western blots were performed using anti-PSer491 antibodies. As shown in Figure 4A, serine 491 was phosphorylated to the same extent in brain extracts from $\mathrm{Cdk5^{+/- }}$ and $\mathrm{Cdk5^{+/+ }}$. In contrast, phosphorylation of this serine was not detected in brain extracts from Cdk5deficient mice, suggesting that in vivo phosphorylation of serine 491 of Dab1 is catalyzed by Cdk5. Because Cdk5-deficient mice exhibit neuronal migration defects similar to those observed in reeler, we asked whether the absence of Cdk5 had any effect on Reelin signaling. Previously, we demonstrated that tyrosine 198 of Dab1 is specifically phosphorylated in response to Reelin (Keshvara et al., 2001). Therefore, we used antibodies specific for this phosphotyrosine (anti-PY198) to investigate Reelin signaling in Cdk5-deficient mice. Immunoblots with anti-PY198 antibodies 
A

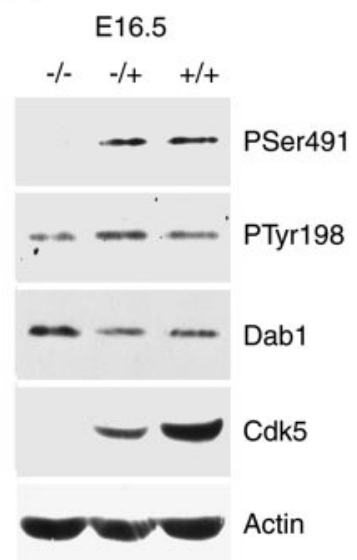

Figure 4. $\mathrm{Cdk} 5$ is required for in vivo phosphorylation of serine 491. Brain lysates prepared from E16.5 $(A)$ or P0 $(B) C d k 5^{-/-}, C d k 5^{-/+}$, or $C d \mathrm{kS}^{+/+}$embryos were immunoprecipitated with anti-Dab1 antibodies. The immunoprecipitates were separated by SDS-PAGE and analyzed by Western blotting using anti-PSer491, anti-PTyr198, 4G10, or anti-Dab1 antibodies. Western blots with anti-C dk5 and anti-actin antibodies were performed on total lysates.

revealed only a modest decrease in phosphorylation of tyrosine 198 in $C d k 5^{-1-}$ mice (Fig. 4A). A characteristic hallmark of a failure in Reelin signaling is the accumulation of Dab1 (Rice et al., 1998). Indeed, we observed a slight increase in Dab1 protein levels in lysates from $C d k 5^{-1-}$ brains (Fig. $4 A$ ). Western analysis with anti-actin antibodies confirmed equal sample loading. Thus, these results suggest that there is a slight but detectable defect in Reelin signaling in $C d k 5^{-/-}$brains at E16.5.

At $\mathrm{P} 0$, the cortical plate is primarily populated by neurons that have completed migration in wild-type mice. To determine whether Reelin signaling is intact at this time in $C d k 5^{-1-}$ mice, we repeated the above experiments using lysates from P0 $C d k 5^{-/-}, C d k 5^{-/+}$, and $C d k 5^{+/+}$brains. As before, we did not observe any phosphorylation of Dab1 on serine 491 in $C d k 5^{-/-}$ mice (Fig. $4 B$ ). In contrast to the situation at E16.5, tyrosine phosphorylation of Dab1 was not detected at P0, suggesting that the Reelin signaling pathway is severely compromised at this age in $C d k 5^{-1-}$ mice. Indeed, we also observed a clear accumulation of Dab1 (threefold) in $C d k 5^{-/-}$mice at P0. In addition, the electrophoretic mobility of Dab1 from $C d k 5^{-1-}$ mice was distinct from that observed in extracts from wild-type mice. This mobility shift was most likely a consequence of hypophosphorylation of Dab1 on both tyrosine and serine residues. Together, these results suggest that, in $C d k 5^{-/-}$mice, the Reelin signaling pathway is intact during the initial stages of cortical plate formation. However, as the brain develops, Reelin signaling is progressively impaired in the absence of Cdk5.

\section{Dab1 levels are elevated in ectopically positioned neurons in the cerebral cortex and cerebellum}

Elevated levels of Dab1 are detected in ectopic neurons in reeler mice (Rice et al., 1998). To determine whether the elevated Dab1 levels detected in $C d k 5^{-1-}$ mice occur specifically in ectopic neurons, we analyzed the distribution of Dab1 in these mice by immunohistochemistry. Immunohistochemistry was performed using rabbit antibodies to Dab1 (B3) on sagittal tissue sections from E18.5 littermates generated by mating $C d k 5^{+/-}$mice. As shown in Figure $5 A$, in wild-type mice, the cortical plate is positioned above the subplate, and it is organized into tightly packed layers of radially oriented neuronal cell bodies. In this case, Dab1 is localized to the cell bodies and the apical dendrites of postmitotic neurons in the cortical plate (Fig. $5 B$ ). In $C d k 5^{-/-}$mice, the cerebral cortex develops into an early cortical plate $(c p)$ separated from an ectopic cortical plate $(e-c p)$ located underneath the subplate $(s p)$ (Fig. $5 D)$. Immunostaining for Dab1 demonstrated that the ectopic cortical plate contained increased levels of Dab1 compared with the normally positioned cortical plate (Fig. 5C). These differences in Dab1 levels were observed at all levels from lateral to dorsal cerebral cortex (data not shown).

In the wild-type cerebellum at E18.5, Purkinje cells migrate to form a Purkinje cell layer $(\mathrm{pcl})$ at superficial positions beneath the external germinal layer $(e g l)$ (Fig. $5 E$ ). Dab1 is expressed in Purkinje cells while they are still migrating (arrows) and in cells that occupy the Purkinje cell layer (Fig. $5 F$ ). Interestingly, the levels of Dab1 are slightly elevated in small populations of Purkinje cells caught in the process of migrating into the Purkinje cell layer (arrows). These populations reside in subcompartments of the developing cerebellum that have been described previously (Nunzi et al., 1999; Ozol et al., 1999). In the $C d k 5^{-1-}$ cerebellum, Purkinje cells fail to migrate out of deep locations, and instead they accumulate in ectopic positions near the neuroepithelium (Fig. $5 H, e-p c)$. Dab1 levels are dramatically elevated in these ectopically located Purkinje cells in the mutant cerebellum (Fig. $5 G)$. Together, these histological studies demonstrate that, in the absence of Cdk5, Dab1 levels are elevated in specific populations of neurons located in ectopic positions in the cerebral cortex and the cerebellum, suggesting that the Reelin signaling pathway is compromised in these cells.

\section{Cdk5-deficient neurons can respond to Reelin}

Because the accumulation of hypophosphorylated Dab1 in $C d k 5^{-1-}$ neurons implies that Reelin signaling is defective, we sought to determine whether the defect in Reelin signaling is intrinsic to $\mathrm{Cdk} 5$-deficient neurons or whether it arises as an indirect consequence of the ectopic location of these neurons. Therefore, neurons isolated from E16.5 $\mathrm{Cdk5^{-/- }}$ and $\mathrm{Cdk5^{+/+ }}$ littermates were treated with Reelin, and changes in Dab1 tyrosine phosphorylation levels were monitored by Western blotting using anti-phosphotyrosine antibodies. As shown in Figure 6, Cdk5-deficient neurons clearly responded to Reelin by exhibiting a sharp increase in tyrosine phosphorylation of Dab1. Western analysis using anti-Dab1 and anti- neuron-specific enolase (NSE) antibodies confirmed equal sample loading. Thus, these results demonstrate that $\mathrm{Cdk} 5$ activity is not required for Reelininduced tyrosine phosphorylation of Dab1.

\section{Serine phosphorylation of Dab1 occurs independently of Reelin}

Because Reelin-induced tyrosine phosphorylation of Dab1 was not affected in $C d k 5^{-1-}$ neurons, we cannot place C $\mathrm{dk} 5$ upstream of Reelin in a linear signaling pathway. Therefore, we investigated the possibility that Cdk5-mediated serine phosphorylation of Dab1 lies downstream of Reelin. Western analysis, using anti-phosphotyrosine and anti-Pser491 antibodies, was performed on Dab1 immunoprecipitated from brain extracts prepared from E14.5 reeler or wild-type embryos. As observed previously, Dab1 levels were significantly elevated in reeler brains, whereas tyrosine phosphorylation was barely detectable (Rice et al., 1998; Howell et al., 1999a) (Fig. 7A). In contrast, there was no apparent change 

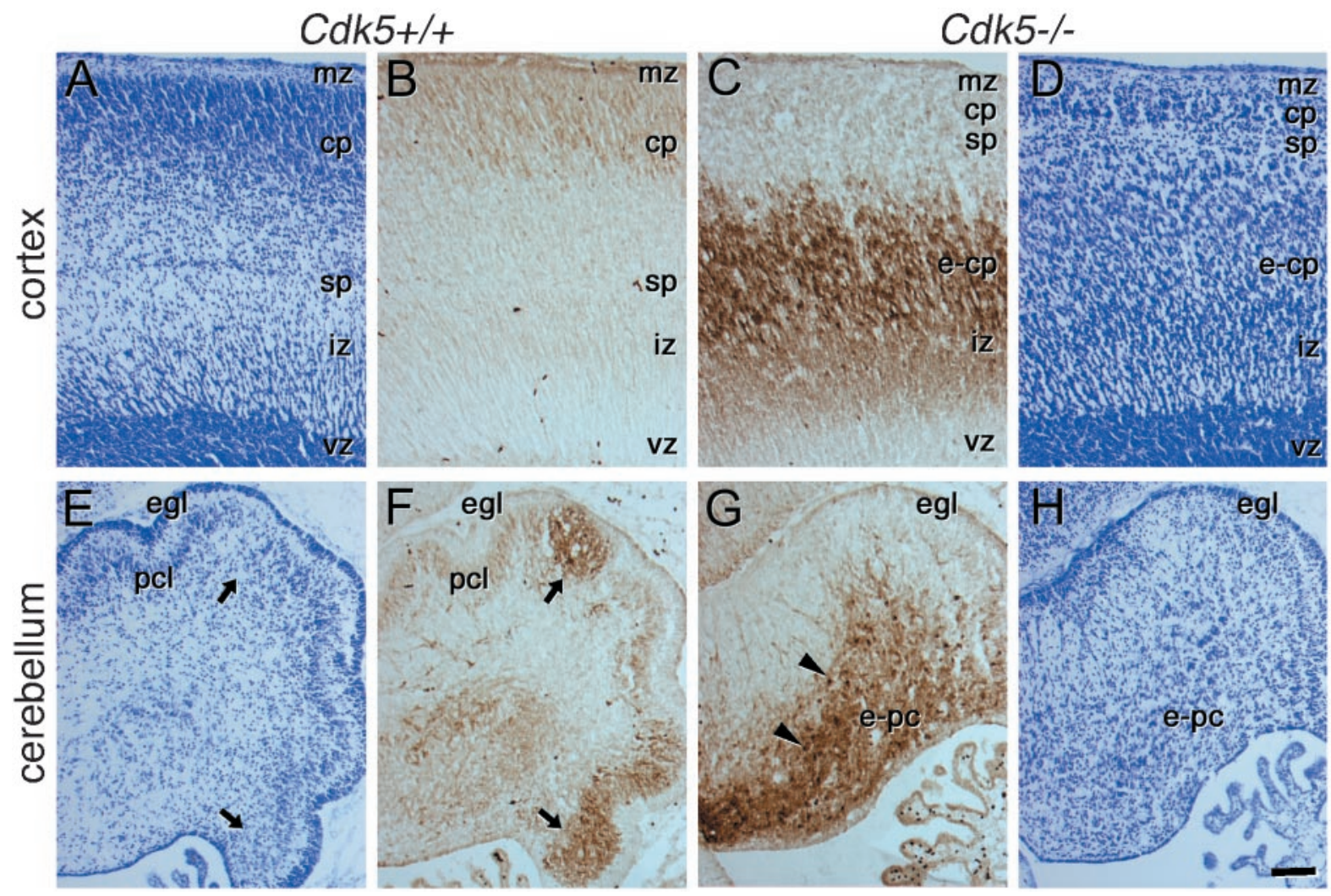

Figure 5. Dab1 is elevated in ectopic neurons in the cerebral cortex and cerebellum. Immunohistochemistry was performed on cortical and cerebellar tissue sections from E18.5 $C d k 5^{+/+}(A, B, E, F)$ and $C d k 5^{-/-}(C, D, G, H)$ mice. $A$, Cresyl violet staining of $C d k 5^{+/+}$mice was used to visualize the normal arrangement of neurons in the cerebral cortex. $B$, In $C d k 5^{+/+}$mice, Dab1 is present in the cell bodies and within the dendrites of cortical plate $(c p)$ neurons. Low or no immunostaining was observed in the subplate $(s p)$ and intermediate zone $(i z)$. $D$, In the $C d k 5^{-/-}$cerebral cortex, a thin cortical plate $(c p)$ forms above a subplate $(s p)$, and neurons accumulate beneath the subplate in an ectopic cortical plate $(e-c p)$. $C$, Dab1 levels appear elevated in neurons that occupy the ectopic cortical plate $(e-c p)$, located beneath the subplate. $E$, Cresyl violet staining of the cerebellum in $C d k 5^{+/+}$mice shows the location of the Purkinje cell layer $(p c l)$ and the external germinal layer $(e g l)$. F, Dab1 is present at detectable levels in Purkinje cells aligned in the Purkinje cell layer (arrows in $E$ and $F$ ). $H$, In the $C d k 5^{-/-}$cerebellum, the ectopic Purkinje cells (e-pc) fail to form a Purkinje cell layer and instead accumulate close to the ventricle as shown by cresyl violet staining. $G$, Dab1 is dramatically elevated in the ectopic Purkinje cells (arrowheads). Digital images were captured at identical settings in $B$ and $C$ and in $F$ and $G$. Scale bar (in $H$ ), $\sim 100 \mu \mathrm{m}$. $m z$, Marginal zone; $v z$, ventricular zone.

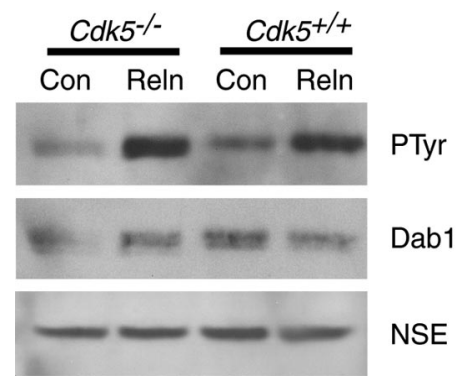

Figure 6. $\mathrm{Cdk} 5$ is not required for Reelin-induced tyrosine phosphorylation of Dab1. Neurons isolated from E16.5 Cdk5 $5^{-1-}$ or $C d k 5^{+1+} \mathrm{em}-$ bryos were suspended in DMEM alone (Con) or Reelin-enriched DMEM (Reln). Treated neurons were then lysed and immunoprecipitated with anti-Dab1 antibodies. The immunoprecipitates were separated by SDSPAGE and transferred to nitrocellulose, and Western blots were performed using 4G10 anti-phosphotyrosine (PTyr) or anti-Dab1 (Dab1) antibodies. Lysates were also probed with anti-NSE antibodies to confirm equal sample loading.

in the stoichiometry of phosphorylation of serine 491 in reeler. To further examine the effect of Reelin stimulation on serine phosphorylation of Dab1, neurons isolated from E16.5 reeler brains were treated in vitro with exogenous Reelin for $15 \mathrm{~min}$ at $37^{\circ} \mathrm{C}$. As expected, Reelin stimulation resulted in a sharp increase in ty-
A

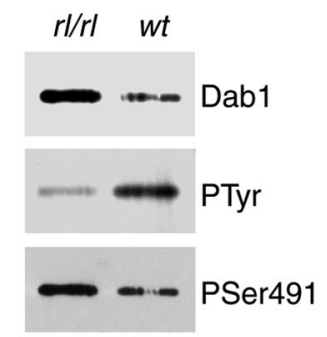

B

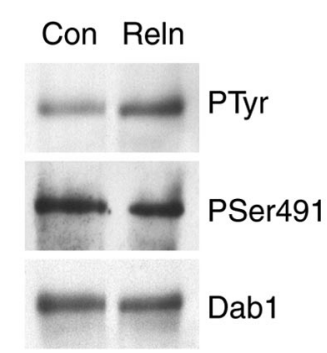

Figure 7. Phosphorylation of Dab1 on serine 491 does not require Reelin signaling. $A$, Brain lysates prepared from E14.5 reeler $(r l / r l)$ or wild-type (wt) littermates were immunoprecipitated with anti-Dab1 antibodies. The immunoprecipitates were then separated by SDS-PAGE, transferred to nitrocellulose, and probed with anti-Dab1, anti-phosphotyrosine, or antiPSer491 antibodies. B, E16.5 reeler neurons were either left untreated (Con) or treated with Reelin (Reln), and phosphorylation was determined by anti-phosphotyrosine and anti-PSer491 antibodies as before.

rosine phosphorylation of Dab1 (Fig. 7B). In contrast, serine 491 was constitutively phosphorylated in these neurons, and exposure to Reelin did not cause any noticeable change. These results suggest that Cdk5-mediated serine phosphorylation of Dab1 occurs independently of Reelin. 


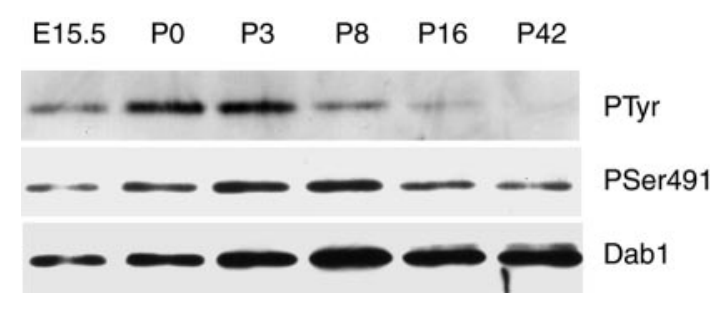

Figure 8. Changes in Dab1 serine and tyrosine phosphorylation during development. Brain lysates were prepared from E15.5, P0, P3, P8, P16, or P42 wild-type mice. Anti-Dab1 immunoprecipitates from these lysates were separated by SDS-PAGE and transferred to nitrocellulose. The membrane was sequentially probed with anti-phosphotyrosine, antiPSer491, and anti-Dab1 antibodies.

\section{Phosphorylation of Dab1 during development}

To gain some understanding of the role of Cdk5-mediated serine phosphorylation of Dab1 during the course of brain development, we compared serine and tyrosine phosphorylation of Dab1 in E15.5, P0, P3, P8, P16, and P42 brains. As shown in Figure 8., Dab1 was phosphorylated on tyrosine only in the embryonic and early postnatal brains, and no significant tyrosine phosphorylation of Dab1 was observed after postnatal day 16. In contrast, phosphorylation of serine 491 was present in both embryonic and adult tissue. Indeed, serine phosphorylation of Dab1 closely matched the pattern of Cdk5 expression and activity, which has been shown to be very low during embryogenesis but to peak in the adult (Tsai et al., 1993).

\section{DISCUSSION}

Reelin and Cdk5 have emerged as critical components of two signaling pathways that regulate neuronal migration during mammalian brain development (Rice and Curran, 2001). Mice with disruptions in reelin, dab1, or both vldlr and apoER2 exhibit the reeler phenotype, which led to the placement of these molecules within a signaling pathway (D'Arcangelo et al., 1995; Howell et al., 1997b; Sheldon et al., 1997; Trommsdorff et al., 1999). The lamination defects observed in mice deficient in Cdk5 (Ohshima et al., 1996; Gilmore et al., 1998; Ohshima et al., 1999), p35 (Kwon and Tsai, 1998), or both p35 and p39 (Ko et al., 2001) are less severe but similar to those found in reeler, suggesting that there may be a cross-talk between the Reelin and Cdk5 pathways. Therefore, we hypothesized that such a biochemical link between the two pathways might involve direct phosphorylation of Dab1 by Cdk5. Here we show that Cdk5 phosphorylates Dab1 on serine 491 in vivo.

Our findings present an interesting possibility that Dab1 may serve as a point of convergence for the Reelin and Cdk5 pathways. Dab1 contains several features of an adapter molecule that may be involved in more than one pathway. The PTB domain within its N-terminal region anchors Dab1 to NPxY sequence motifs present within the cytoplasmic domains of various receptors, including the lipoprotein receptors VLDLR and ApoER2 (Trommsdorff et al., 1998) and the amyloid peptide precursor family of proteins (Homayouni et al., 1999; Howell et al., 1999b). Dab1 contains several tyrosine residues that are critical for its function in the Reelin pathway (Howell et al., 2000), and at least two of these tyrosines are phosphorylated in response to Reelin stimulation (Keshvara et al., 2001). Reelin-induced phosphorylation of these tyrosines is likely to result in creation of docking sites on Dab1 for recruitment of signaling molecules containing Src homology 2 (SH2) domains (Songyang et al., 1993). At least in vitro, tyrosine phosphorylated Dab1 has been shown to interact with SH2 domain-containing proteins (Howell et al., 1997a). Therefore, Dab1 is likely to serve as a scaffold for assembly of a signaling complex that would couple an upstream signal from Reelin to the molecular machinery involved in cell positioning. Cdk5-mediated serine phosphorylation could potentially modulate the function of the Dab1-associated signaling complex. For example, it is possible that phosphorylated serine residues could serve to recruit additional signaling proteins containing phosphoserine protein-interaction modules, similar to the phosphoserine binding exhibited by 14-3-3 proteins (Yaffe and Elia, 2001). Alternatively, phosphorylation on serine may induce a conformational change in Dab1 that might influence its interaction with downstream effector molecules.

Unlike tyrosine phosphorylation of Dab1, phosphorylation of serine 491 by Cdk5 occurs independently of the presence of Reelin. Therefore, Reelin is unlikely to play a role in regulating Cdk5 activity. Conversely, Cdk5-deficient neurons exhibit a normal Reelin response, whereby Dab1 is tyrosine phosphorylated as a result of exposure to Reelin. Therefore, Cdk5-mediated serine phosphorylation of Dab1 is not required for the Reelin-response. Also, examination of brain lysates from different ages showed that tyrosine phosphorylation of Dab1 is restricted to embryonic and early postnatal brains, whereas serine phosphorylation of Dab1 occurs throughout development and in the adult. Together, these results are consistent with a model in which Reelin and Cdk5 are components of two distinct signaling pathways. Indeed, recent analysis of mice lacking both p35 and Dab1 revealed additive defects in the cerebellum and in the hippocampus, suggesting that neuronal positioning is regulated by contributions from both of these pathways (Ohshima et al., 2001). Nevertheless, a cross-talk between the two pathways, through serine phosphorylation of Dab1, may represent an important mechanism for fine tuning of neuronal migration.

Persistence of serine phosphorylation of Dab1 in adult mice suggests a potential role for $\mathrm{Cdk} 5$-mediated Dab1 phosphorylation in neuronal functions that occur after the completion of cell positioning. Indeed, the Reelin signaling pathway has been implicated in synaptogenesis (Borrell et al., 1999; Rice et al., 2001). Therefore, it is possible that serine phosphorylation of Dab1 may play a role in such post-positioning functions. It is not known whether Dab1 is involved in other cellular processes. However, in light of our results indicating a biochemical link between Cdk5 and Dab1, it will now be important to investigate a potential role for Dab1 in events that require $\mathrm{Cdk} 5$. Cdk5 is thought to contribute to the pathology of neurodegenerative disorders, such as Alzheimer's disease (Patrick et al., 1999) and amyotrophic lateral sclerosis (Nguyen et al., 2001). Cdk5 is also implicated in the regulation of numerous other cellular events, including neurite extension (Nikolic et al., 1996), cell adhesion (Homayouni and Curran, 2000; Kwon et al., 2000), and axonal transport (Niethammer et al., 2000; Sasaki et al., 2000; Wynshaw-Boris and Gambello, 2001). Therefore, given the overlap of the Cdk5 and Dab1 expression patterns, it is possible that Dab1 may also participate in these cellular processes.

Dab1 was found to accumulate in a hypophosphorylated form in $C d k 5^{-1-}$ neurons. Decreased tyrosine phosphorylation of Dab1 and its subsequent accumulation were very slight at E16.5 in $C d k 5^{-1-}$ brains, but these changes became dramatic by $\mathrm{P} 0$. One possible interpretation of this result is that $C d k 5^{-/-}$neurons suffer from an intrinsic biochemical defect that prevents Reelininduced Dab1 tyrosine phosphorylation. However, this is un- 
likely, because cultured $C d k 5^{-1-}$ neurons exhibited a normal Dab1 tyrosine phosphorylation response when treated with exogenous Reelin. When Dab1 expression was analyzed by immunohistochemistry, Dab1 accumulation was particularly prevalent in neurons located in deeper layers of the cerebral cortex and the cerebellum. Previous neuroanatomic analysis of Cdk5- and p35deficient mice demonstrated that the first cohorts of neurons are able to migrate past subplate neurons, but later-born neurons fail to overtake their predecessors (Gilmore et al., 1998; Kwon and Tsai, 1998). These subsequent failures in migration lead to ectopic accumulation of neurons beneath the subplate. Similarly, in the cerebellum, Purkinje cells fail to migrate outward to form the Purkinje cell layer, and instead they are clustered in deep regions of the cerebellum (Ohshima et al., 1999). The accumulation of Dab1 in $C d k 5^{-1-}$ mice is confined to these ectopic neurons arrested deep within the cortex and the cerebellum. The most straightforward interpretation of our results is that, in the absence of C dk5, ectopic neurons fail to reach the source of Reelin, which is expressed in the marginal zone of the cortex and in the external germinal layer and nuclear transitory zone of the cerebellum in the developing brain. This effect becomes progressively more severe at later stages of development when neurons have to traverse greater distances to contact Reelin. Therefore, it is unlikely that the diminished Reelin signaling in $\mathrm{Cdk5^{-/ }}$ neurons during late embryogenesis is attributable to an intrinsic defect in the Reelin pathway within these neurons, but rather it is a consequence of their ectopic location. This hypothesis can be tested by expressing Reelin in locations more proximal to the ectopic neurons of the cerebral cortex and cerebellum in $C d \mathrm{k5}^{-/-}$ mice.

We identified serine 491 of Dab1 as a Cdk5-dependent site of in vivo phosphorylation. However, we cannot rule out the possibility that there are other Cdk5 phosphorylation sites in Dab1. Indeed, our phosphopeptide mapping analysis suggested that Cdk5 also phosphorylates serine 515 of Dab1 in vitro. Nevertheless, antibodies against phosphoserine 491 of Dab1 provide a valuable marker for $\mathrm{Cdk} 5$ activity in vivo, and they can be used to further investigate the role of Cdk5 in neural functions within cells that express Dab1. Although the functional significance of Cdk5-catalyzed phosphorylation of Dab1 is not yet clear, our results suggest that Reelin and $\mathrm{Cdk} 5$ are components of two distinct signaling pathways that may converge on Dab1. Cdk5mediated serine phosphorylation of Dab1 may serve to modulate signaling events initiated by Reelin that lie downstream of Dab1 tyrosine phosphorylation. Alternatively, serine phosphorylation might point to a potential role for Dab1 beyond the Reelin signaling pathway. A comprehensive approach involving a combination of genetic and biochemical techniques will now be required to clarify the biochemical and neuroanatomical consequences of Cdk5-mediated serine phosphorylation of Dab1.

\section{REFERENCES}

Borrell V, Del Rio JA, Alcantara S, Derer M, Martinez A, D'Arcangelo G, Nakajima K, Mikoshiba K, Derer P, Curran T, Soriano E (1999) Reelin regulates the development and synaptogenesis of the layerspecific entorhino-hippocampal connections. J Neurosci 19:1345-1358.

Chae T, Kwon YT, Bronson R, Dikkes P LiE, Tsai LH (1997) Mice lacking $\mathrm{p} 35$, a neuronal specific activator of $\mathrm{Cdk} 5$, display cortical lamination defects, seizures, and adult lethality. Neuron 18:29-42.

D'Arcangelo G, Curran T (1998) Reeler: new tales on an old mutant mouse. BioEssays 20:235-244.

D'Arcangelo G, Miao GG, Chen SC, Soares HD, Morgan JI, Curran T (1995) A protein related to extracellular matrix proteins deleted in the mouse mutant reeler. Nature 374:719-723.

D’Arcangelo G, Homayouni R, Keshvara L, Rice DS, Sheldon M, Curran
$\mathrm{T}$ (1999) Reelin is a ligand for lipoprotein receptors. Neuron 24:471-479.

Dhavan R, Tsai LH (2001) A decade of cdk5. Nat Rev Mol Cell Biol 2:749-759.

Gilmore EC, Ohshima T, Goffinet AM, Kulkarni AB, Herrup K (1998) Cyclin-dependent kinase 5-deficient mice demonstrate novel developmental arrest in cerebral cortex. J Neurosci 18:6370-6377.

Hiesberger T, Trommsdorff M, Howell BW, Goffinet A, Mumby MC, Cooper JA, Herz J (1999) Direct binding of Reelin to VLDL receptor and ApoE receptor 2 induces tyrosine phosphorylation of disabled-1 and modulates tau phosphorylation. Neuron 24:481-489.

Homayouni R, Curran T (2000) Cortical development: Cdk5 gets into sticky situations. Curr Biol 10:R331-R334.

Homayouni R, Rice DS, Sheldon M, Curran T (1999) Disabled-1 binds to the cytoplasmic domain of amyloid precursor-like protein 1 . J Neurosci 19:7507-7515.

Howell BW, Gertler FB, Cooper JA (1997a) Mouse disabled (mDab1): a Src binding protein implicated in neuronal development. EMBO J $16: 121-132$.

Howell BW, Hawkes R, Soriano P, Cooper JA (1997b) Neuronal position in the developing brain is regulated by mouse disabled-1. Nature 389:733-737.

Howell BW, Herrick TM, Cooper JA (1999a) Reelin-induced tryosine phosphorylation of disabled 1 during neuronal positioning. Genes Dev 13:643-648.

Howell BW, Lanier LM, Frank R, Gertler FB, Cooper JA (1999b) The disabled 1 phosphotyrosine-binding domain binds to the internalization signals of transmembrane glycoproteins and to phospholipids. Mol Cell Biol 19:5179-5188.

Howell BW, Herrick TM, Hildebrand JD, Zhang Y, Cooper JA (2000) Dab1 tyrosine phosphorylation sites relay positional signals during mouse brain development. Curr Biol 10:877-885.

Keshvara L, Benhayon D, Magdaleno S, Curran T (2001) Identification of reelin-induced sites of tyrosyl phosphorylation on disabled 1. J Biol Chem 276:16008-16014.

Ko J, Humbert S, Bronson RT, Takahashi S, Kulkarni AB LiE, Tsai LH (2001) p35 and p39 are essential for cyclin-dependent kinase 5 function during neurodevelopment. J Neurosci 21:6758-6771.

Kwon YT, Tsai LH (1998) A novel disruption of cortical development in p35(-/-) mice distinct from reeler. J Comp Neurol 395:510-522.

Kwon YT, Gupta A, Zhou Y, Nikolic M, Tsai LH (2000) Regulation of $\mathrm{N}$-cadherin-mediated adhesion by the p35-Cdk5 kinase. Curr Biol $10: 363-372$

Lew J, Huang QQ QiZ, Winkfein RJ, Aebersold R, Hunt T, Wang JH (1994) A brain-specific activator of cyclin-dependent kinase 5. Nature 371:423-426.

Nguyen MD, Lariviere RC, Julien JP (2001) Deregulation of Cdk5 in a mouse model of ALS: toxicity alleviated by perikaryal neurofilament inclusions. Neuron 30:135-147.

Niethammer M, Smith DS, Ayala R, Peng J Ko J, Lee MS, Morabito M, Tsai LH (2000) NUDEL is a novel Cdk5 substrate that associates with LIS1 and cytoplasmic dynein. Neuron 28:697-711.

Nikolic M, Dudek H, Kwon YT, Ramos YF, Tsai LH (1996) The cdk5/ p35 kinase is essential for neurite outgrowth during neuronal differentiation. Genes Dev 10:816-825.

Nunzi MG, Grillo M, Margolis FL, Mugnaini E (1999) Compartmental organization of Purkinje cells in the mature and developing mouse cerebellum as revealed by an olfactory marker protein-lac Z transgene. J Comp Neurol 404:97-113.

Ohshima T, Ward JM, Huh CG, Longenecker G, Veeranna, Pant HC, Brady RO, Martin LJ, Kulkarni AB (1996) Targeted disruption of the cyclin-dependent kinase 5 gene results in abnormal corticogenesis, neuronal pathology and perinatal death. Proc Natl Acad Sci USA 93:11173-11178.

Ohshima T, Gilmore EC, Longenecker G, Jacobowitz DM, Brady RO, Herrup K, Kulkarni AB (1999) Migration defects of cdk5(-/-) neurons in the developing cerebellum is cell autonomous. J Neurosci 19:6017-6026.

Ohshima T, Ogawa M, Veeranna, Hirasawa M, Longenecker G, Ishiguro K, Pant HC, Brady RO, Kulkarni AB, Mikoshiba K (2001) Synergistic contributions of cyclin-dependant kinase 5/p35 and Reelin/Dab1 to the positioning of cortical neurons in the developing mouse brain. Proc Natl Acad Sci USA 98:2764-2769.

Ozol K, Hayden JM, Oberdick J, Hawkes R (1999) Transverse zones in the vermis of the mouse cerebellum. J Comp Neurol 412:95-111.

Patrick GN, Zukerberg L, Nikolic M, de la Monte S, Dikkes P, Tsai LH (1999) Conversion of p35 to p25 deregulates Cdk5 activity and promotes neurodegeneration. Nature 402:615-622.

Rice DS, Curran T (2001) Role of the Reelin signaling pathway in central nervous system development. Annu Rev Neurosci 24:1005-1039.

Rice DS, Sheldon M, D'Arcangelo G, Nakajima K, Goldowitz D, Curran $\mathrm{T}$ (1998) Disabled-1 acts downstream of Reelin in a signaling pathway that controls laminar organization in the mammalian brain. Development 125:3719-3729. 
Rice DS, Nusinowitz S, Azimi AM, Martinez A, Soriano E, Curran T (2001) The reelin pathway modulates the structure and function of retinal synaptic circuitry. Neuron 31:929-941.

Sasaki S, Shionoya A, Ishida M, Gambello MJ, Yingling J, WynshawBoris A, Hirotsune S (2000) A LIS1/NUDEL/cytoplasmic dynein heavy chain complex in the developing and adult nervous system. Neuron 28:681-696.

Sheldon M, Rice DS, D'Arcangelo G, Yoneshima H, Nakajima K, Mikoshiba K, Howell BW, Cooper JA, Goldowitz D, Curran T (1997) Scrambler and yotari disrupt the disabled gene and produce a reelerlike phenotype in mice. Nature 389:730-733.

Songyang Z, Shoelson SE, Chaudhuri M, Gish G, Pawson T, Haser WG, King F, Roberts T, Ratnofsky S, Lechleider RJ, Neel BG, Birge RB, Fajardo JE, Chou MM, Hanafusa H, Schaffhausen B, Cantley LC (1993) SH2 domains recognize specific phosphopeptide sequences. Cell $72: 767-778$

Songyang Z, Lu KP, Kwon YT, Tsai LH, Filhol O, Cochet C, Brickey DA, Soderling TR, Bartleson C, Graves DJ, DeMaggio AJ, Hoekstra MF, Blenis J, Hunter T, Cantley LC (1996) A structural basis for substrate specificities of protein Ser/Thr kinases: primary sequence preference of casein kinases I, II, NIMA, phosphorylase kinase, calmodulin-dependent kinase II, CDK5, and Erk1. Mol Cell Biol 16:6486-6493.

Tang D, Yeung J, Lee KY, Matsushita M, Matsui H, Tomizawa K, Hatase O, Wang JH (1995) An isoform of the neuronal cyclin-dependent kinase 5 (Cdk5) activator. J Biol Chem 270:26897-26903.

Trommsdorff M, Borg JP, Margolis B, Herz J (1998) Interaction of cytosolic adaptor proteins with neuronal apolipoprotein $\mathrm{E}$ receptors and the amyloid precursor protein. J Biol Chem 273:33556-33560.

Trommsdorff M, Gotthardt M, Hiesberger T, Shelton J, Stockinger W, Nimpf J, Hammer RE, Richardson JA, Herz J (1999) Reeler/ Disabled-like disruption of neuronal migration in knockout mice lacking the VLDL receptor and ApoE receptor 2. Cell 97:689-701.

Tsai LH, Takahashi T, Caviness Jr VS, Harlow E (1993) Activity and expression pattern of cyclin-dependent kinase 5 in the embryonic mouse nervous system. Development 119:1029-1040.

Tsai LH, Delalle I, Caviness Jr VS, Chae T, Harlow E (1994) p35 is a neural-specific regulatory subunit of cyclin-dependent kinase 5 . Nature 371:419-423.

Wynshaw-Boris A, Gambello MJ (2001) LIS1 and dynein motor function in neuronal migration and development. Genes Dev 15:639-651.

Yaffe MB, Elia AE (2001) Phosphoserine/threonine-binding domains. Curr Opin Cell Biol 13:131-138. 